\title{
A solvable blob-model for magnetized plasmas
}

\author{
H. L. Pécseli ${ }^{1}$, D. S. Sortland ${ }^{2}$, and O. E. Garcia ${ }^{2}$ \\ ${ }^{1}$ University of Oslo, Physics Department, P.O. Boks 1048 Blindern, N-0316 Oslo, \\ Norway \\ ${ }^{2}$ Department of Physics and Technology, UiT The Arctic University of Norway, \\ N-9019 Troms $\varnothing$, Norway \\ E-mail: hans.pecseli@fys.uio.no \\ E-mail: daniel.s.sortland@uit.no \\ E-mail: odd.erik.garcia@uit.no
}

\begin{abstract}
A simple analytically solvable model for blobs in magnetized plasmas is proposed. The model gives results for a scaling of the blob velocity with the amplitude of the density perturbation. Limiting cases are considered: one where the plasma motion is strictly perpendicular to an externally imposed toroidal magnetic field, and one where the electrons can move along magnetic field lines to compensate partly the collective electric fields. For these limiting cases, the model predicts scaling laws for the dependence of the blob velocities and accelerations with varying cross section, plasma density and temperature. Also the scaling with the dominant ion mass is derived. The analysis is completed by including the effects of collisions between ions and neutrals.
\end{abstract}

PACS numbers: 52.25.Xz, 52.25.Fi, 52.20.Dq 


\section{Introduction}

22 The most effective mixing agency in neutral atmospheres is turbulence. Qualitatively, 23 this process can be described as a random walk mediated by turbulent eddies [1]. 24 Turbulent transport in this sense is found also in laboratory plasma experiments, fusion 25 related studies in particular [2]. In a number of cases it turns out, however, that 26 the anomalous plasma losses across magnetic field lines are due to propagating large 27 structures that appear randomly distributed in space and time [3]. In some cases these 28 structures span large parts of the main plasma and appear as "streamers" $[4,5]$. In other 2 cases the structures are best described as individual "blobs" that can become detached so from the main plasma and propagate towards the walls of the plasma confining vessel $31[6,7,8,9,10,11,12,13,14]$. Such models were found useful also for modeling random 32 plasma signals and probability densities [5].

The properties of individual plasma blobs have been studied in detail by a combination of numerical and analytical models [15], often using some prescribed analytical spatial form, for instance an initial Gaussian shape that subsequently evolves in time. Analytical results, supported by numerical simulations predict, for instance, a "blob velocity" perpendicular to magnetic field lines. In the small density perturbation limit, $\Delta n / n \ll 1$, the velocity scaling is

$$
U \sim \sqrt{R_{b} \frac{\Delta n}{n}},
$$

where $2 R_{b}$ is the filament or blob width in the direction perpendicular to the local magnetic field $\mathbf{B}$. For large $\Delta n / n$, the velocity saturates $[6,15]$ and becomes nearly independent of $\Delta n / n$. A summary for blob velocity models can be found in the literature [13]. The results from the present study can serve as a useful reference or test-case for other more elaborate models. Models of individual blob structures will in general be quite complicated, and a simple solvable model have some advantages for discussing basic properties. Such a model is suggested here by assuming a circular "top-hat" density variation of the plasma density, i.e. the plasma density is $n_{0}$ inside a circular cross section and vanishes outside. With the steep gradients at the edges of the blobs in the present model we can not assume quasi-neutrality and the internal electric fields have to be determined from the charge separations. One feature of these top-hat models is to demonstrate that a scaling like (1) is model dependent, and thus not universal. Another feature of the present model is a limiting case where blobs move not with constant velocity, but constant acceleration in the major radius direction of the torus. The acceleration is found to be independent of the blob width perpendicular to the magnetic field, at least as long as this scale is much larger than the ion gyro radius, $r_{L i}$. When $R_{b}$ is comparable to $r_{L i}$, the acceleration becomes smaller due to the spatial averaging $[16,17]$ of the electric fields associated with the blobs.

The present study is organized as follows. In Section 2 we describe a simple model for polarization of a cylindrical form. For the assumed slow dynamics with variations on a time scale much larger than the ion gyro-time $M / e B \equiv \Omega_{c i}^{-1}$, where $\Omega_{c i}$ is the 
ion gyro frequency, we have the dominant plasma polarization being due to the ion polarization drifts. The analysis assumes a toroidal geometry for the magnetic field. In this case the ions move across magnetic field lines due to curvature and magnetic gradient drifts [16]. The basic model allows a simple generalization to magnetized plasmas in gravitational fields as discussed in Section 3. Some straight forward extensions of these results are discussed in Section 4. The simplest model assumes that both the dominant electron and the ion motions are strictly perpendicular to the local magnetic field B. In Section 5.1 we relax this restriction on the electron dynamics and use a mixed plasma model analogous to what is known as the Hasegawa-Wakatani model [18], where the dominant ion motion remains perpendicular to $\mathbf{B}$, but the electrons move along magnetic field lines, subject to a collisional drag, due to for instance collisions with a neutral background. Section 5.2 includes collisional friction in the ion dynamics. Finally,

Section 7 contains our conclusions.

\section{A simple analytical model for blob polarization by $\nabla|\mathrm{B}|$ drifts}

With the present model we include the spatial variation of the magnetic field. For a toroidal geometry we find $|\mathbf{B}|=B_{0} R_{0} / R$ where $R$ is the major radial position in the torus and $R_{0}$ is a reference position in the center of the toroidal cross section. For this case we have $|\nabla B|=B_{0} R_{0} / R^{2}$. In the vicinity of the central position $R_{0}$, the $\nabla B$ ion drift velocity averaged over a thermal particle population becomes

$$
U_{\nabla B}=\frac{1}{2} \frac{M u_{t h i}^{2}}{e B^{2}}|\nabla B|=\frac{1}{2} \frac{M u_{t h i}^{2}}{e B_{0} R_{0}},
$$

with $u_{t h i}^{2}=T_{i} / M$ being the ion thermal velocity. If we include also the curvature drift for a particle population in thermal equilibrium [16] we find a simple modification of this result to give

$$
U_{i}=\frac{3}{2} \frac{M u_{t h i}^{2}}{e B^{2}}|\nabla B|=\frac{3}{2} \frac{M u_{t h i}^{2}}{e B_{0} R_{0}} .
$$

The corresponding expressions for the electron drifts are found be the replacements $e \rightarrow-e, M \rightarrow m$ and $T_{i} \rightarrow T_{e}$. It can be demonstrated $[16,19]$ that the $\nabla B$ ion drift and the curvature drift velocities are in general additive for low- $\beta$ plasmas where $\nabla \times \mathbf{B}=0$.

We consider a circular cross section of a blob-structure with a uniform density $n_{0}$. The radius of the circular cross section turns out to be of minor importance for details in the analysis. We assume the space-time varying plasma density to be strictly toroidally aligned at all times. The $\nabla B$-velocity caused by the inhomogeneous magnetic field is constant and in the $\widehat{\mathbf{z}}$-direction. The $\nabla B$ electron and ion drifts polarize the blob and the polarization charges give rise to an electric field $\mathbf{E}(\mathbf{r}, t)$. In the moving frame of reference we have in addition to $\mathbf{E}$ an induced electric field due to the plasma motion across magnetic field lines. We take this additional field to be $-\mathbf{U}_{i, e} \times \mathbf{B} \equiv-d \mathbf{R}_{i, e} / d t \times \mathbf{B}$, respectively for ions and electrons, as in ideal magneto hydrodynamics. Since the blobs 
will be accelerated in general, the moving frame is in not always an inertial frame of reference, and the exact transformation will be more complicated.

The basic equation of motion for the center-of-mass $\mathbf{R}_{i}(t)$ of the ion component is then

$$
\frac{d}{d t} \mathbf{R}_{i}(t)=\frac{\mathbf{E}\left(\mathbf{R}_{i}(t), t\right) \times \mathbf{B}}{B^{2}}+\frac{1}{\Omega_{c i}} \frac{d}{d t} \frac{\mathbf{E}\left(\mathbf{R}_{i}(t), t\right)}{B}+U_{i} \widehat{\mathbf{z}}
$$

Through the ion cyclotron frequency $\Omega_{c i}$, the ion mass appears explicitly due to inclusion of the ion polarization drift. A collisional drag on the ions was ignored here, to be discussed in the following Section 5.2.

An expression similar to (2) for the electron dynamics becomes

$$
\frac{d}{d t} \mathbf{R}_{e}(t)=\frac{\mathbf{E}\left(\mathbf{R}_{e}(t), t\right) \times \mathbf{B}}{B^{2}}-U_{e} \widehat{\mathbf{z}} .
$$

In general we have $\left|U_{i}\right| \neq\left|U_{e}\right|$ because of different ion and electron temperatures. Note that the electric fields in (2) and (3) are to be obtained at $\mathbf{R}_{i}(t)$ and $\mathbf{R}_{e}(t)$, respectively, so the two terms need not cancel at subtraction of the two expressions. The spatial variation of the magnetic field is included via the last terms in (2) and (3). It is an essential feature of the model that an initially circular contour will remain circular at all later times since $U_{i}$ and $U_{e}$, as well as the $\mathbf{E} \times \mathbf{B} / B^{2}$ velocities are taken spatially constant inside the blob.

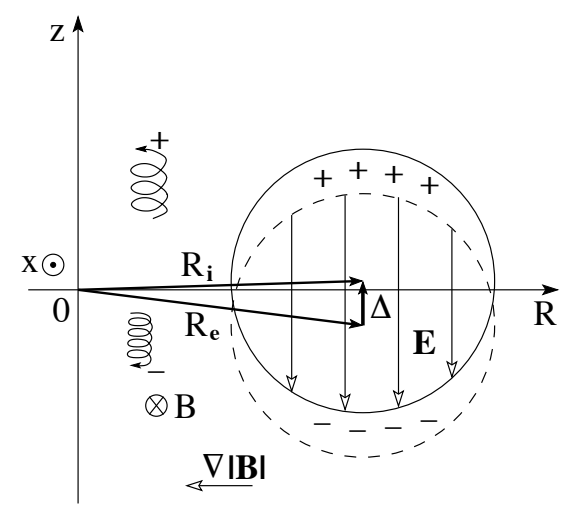

Figure 1. Schematic illustration of the polarization of a simple model here with a circular cross section and uniform density. The $\nabla B$ direction as well as illustrative ion and electron $\nabla B$-drifts are shown for reference. The magnetic field vector points into the plane of the figure. The $z$-axis is also the symmetry axis for the torus. The magnetic field is here taken anti-parallel to the $x$-axis perpendicular to the plane of the figure. The components of the vectors $\mathbf{R}_{i, e}$ are expressed in terms of coordinates $(R, z)$.

The electric field originates from time varying part induced by polarization of the plasma. With $-e$ being the electron charge we find

$$
\mathbf{E}=-\frac{1}{2} \frac{e n_{0}}{\varepsilon_{0}} \boldsymbol{\Delta}
$$


where $\boldsymbol{\Delta}(t) \equiv \mathbf{R}_{i}(t)-\mathbf{R}_{e}(t)$ where we will assume $|\boldsymbol{\Delta}| \ll\left|\mathbf{R}_{i, e}\right|$ as well as $|\boldsymbol{\Delta}| \ll R_{b}$. The vectors $\mathbf{R}_{i}, \mathbf{R}_{e}$ and $\boldsymbol{\Delta}$ are explained in Fig. 1. The magnitude of the displacement vector $|\boldsymbol{\Delta}|$ is assumed to be much smaller than $R_{0}$.

Surface charges are created when the electrons are displaced slightly with respect to the ions. It is well known that these charges give rise to a constant electric field inside the central lens-shaped part of the cross section, see Fig. 1, with the field direction being along $-\boldsymbol{\Delta}$. The factor $1 / 2$ in (4) originates from the locally cylindrical geometry. Throughout in the following we assume that $|\Delta| \ll R_{e, i}$. We introduce the blob radius as $R_{b}$. The analytical variation for the electrostatic potential in the fixed frame for is $\phi \sim r \sin \theta$ or $\phi \sim z$ in Cartesian coordinates, while outside the blob we have $\phi \sim \frac{1}{r} \sin \theta$ or $\phi \sim z /\left(R^{2}+z^{2}\right)$. Inside the "top-hat" blob we have a constant electric field. For the electric field components outside the blob we have

$$
E_{R} \sim-\frac{R^{2}-z^{2}}{\left(R^{2}+z^{2}\right)^{2}} \quad \text { and } \quad E_{z} \sim \frac{2 R z}{\left(R^{2}+z^{2}\right)^{2}},
$$

in terms of the coordinates defined in Fig. 1. An illustration of the electric field vectors is given in Fig. 2.

We can write the equation of motion for the ion center of mass as

$$
\begin{aligned}
& \frac{d \mathbf{R}_{i}(t)}{d t}= \\
& \quad-\frac{1}{2} \Omega_{c i}\left(\varepsilon_{r}-1\right) \boldsymbol{\Delta}(t) \times \widehat{\mathbf{b}}-\frac{1}{2}\left(\varepsilon_{r}-1\right) \frac{d \boldsymbol{\Delta}(t)}{d t}+U_{i} \widehat{\mathbf{z}},
\end{aligned}
$$

and for the electrons

$$
\frac{d \mathbf{R}_{e}(t)}{d t}=-\frac{1}{2} \Omega_{c i}\left(\varepsilon_{r}-1\right) \boldsymbol{\Delta}(t) \times \widehat{\mathbf{b}}-U_{e} \widehat{\mathbf{z}}
$$

where electron polarization drifts are ignored. The relative dielectric plasma constant $\varepsilon_{r} \equiv 1+n_{0} M / \varepsilon_{0} B^{2}=1+\left(\Omega_{p i} / \Omega_{c i}\right)^{2}$ was also introduced [20]. We introduced the ion plasma frequency so that $\Omega_{p i}^{2} \equiv e^{2} n_{0} /\left(\varepsilon_{0} M\right)$. The present analysis can be made identical to a single particle model because the plasma motion is adequately represented by the center-of-mass of the blob which can be accounted for by the motion of a single particle. This is a considerable simplification compared to a complete fluid model [19].

The spatial variation of $\epsilon_{r}$ through the spatial variation of $\mathbf{B}$ is ignored by making a local analysis. The spatial variation of $B$ enters only through the $\nabla B$-drift. Due to the "top hat" model we have the plasma density to be constant inside the structure.

Subtracting (2) and (3) we obtain an ordinary differential equation for $\boldsymbol{\Delta}(t)=$ $\mathbf{R}_{i}(t)-\mathbf{R}_{e}(t)$ in the form

$$
\frac{d}{d t} \boldsymbol{\Delta}(t)=\frac{U_{i}+U_{e}}{1+\frac{1}{2} \frac{n_{0} M}{\varepsilon_{0} B^{2}}} \widehat{\mathbf{z}} \equiv 2 \frac{U_{i}+U_{e}}{1+\varepsilon_{r}} \widehat{\mathbf{z}} .
$$

With the present simplified assumptions, the relative displacement of electrons and ions increases without limit, $|\boldsymbol{\Delta}(t)| \rightarrow \infty$, while the electric fields produced by the 
separation accelerates the blob in the direction of the major radius of the torus. To find the acceleration of the bulk plasma-blob we use the average position $\mathbf{R}_{p}(t) \equiv$ $\left(\mathbf{R}_{i}(t)+\mathbf{R}_{e}(t)\right) / 2$. By adding (5) and (6) we have

$$
\begin{aligned}
& 2 \frac{d \mathbf{R}_{p}}{d t}=-\Omega_{c i}\left(\varepsilon_{r}-1\right) \boldsymbol{\Delta}(t) \times \widehat{\mathbf{b}}+\left(U_{i}-U_{e}\right) \widehat{\mathbf{z}} \\
& \quad-\frac{1}{2}\left(\varepsilon_{r}-1\right) \frac{d \boldsymbol{\Delta}(t)}{d t},
\end{aligned}
$$

which ignores terms of the order of $m / M$ by ignoring the electron polarization drift.

By differentiation of (7) we find

$$
\frac{d^{2} \mathbf{R}_{p}}{d t^{2}}=\Omega_{c i}\left(U_{i}+U_{e}\right) \frac{\varepsilon_{r}-1}{\varepsilon_{r}+1} \widehat{\mathbf{R}},
$$

since $d\left(U_{i}-U_{e}\right) / d t=0$ as well as $d^{2} \boldsymbol{\Delta} / d t^{2}=0$, while $\widehat{\mathbf{z}} \times \widehat{\mathbf{b}}=-\widehat{\mathbf{R}}$ with $z$ and $R$ defined in Fig. 1. We have in particular

$$
\lim _{n_{0} \rightarrow \infty} \frac{d^{2} \mathbf{R}_{p}}{d t^{2}}=\Omega_{c i}\left(U_{i}+U_{e}\right) \widehat{\mathbf{R}}=\text { const. }
$$

For large densities $n_{0}$, i.e. $\Omega_{p i} \gg \Omega_{c i}$, we have $\varepsilon_{r} \sim n_{0}$. In the limiting case for large $n_{0}$ we consequently find that $d^{2} \mathbf{R}_{p} / d t^{2}$ is independent of blob density as indicated in (9). We have a linear scaling with plasma temperature $T_{i, e}$ through $U_{i, e}$. Since $U_{i}$ is independent of the ion mass, we have an inverse scaling of (9) with respect to $M$; heavy ions experience a smaller acceleration than lighter ones. The blob is lost at a constant acceleration in the direction of the major radius, here the positive $\widehat{\mathbf{R}}$-direction, see Fig. 1. This result accounts also for the well known lack of equilibrium for a simple magnetized toroidal plasma [16, 21, 22], since an entire toroidal plasma can also be considered as one large blob.

For low density plasmas, with $\epsilon_{r} \rightarrow 1$ so that $\left(\varepsilon_{r}-1\right) /\left(\varepsilon_{r}+1\right) \approx \frac{1}{2}\left(\varepsilon_{r}-1\right)$, we find $d^{2} \mathbf{R}_{p} / d t^{2} \approx \frac{1}{2} \Omega_{p i}^{2}\left(U_{i}+U_{e}\right) \widehat{\mathbf{R}} / \Omega_{c i}$ which scales as $\sim n_{0} T$, being independent of ion mass. Lower density blobs are lost at a slower rate than those with high density. A qualitative argument then gives that the cross section of a blob with inhomogeneous density (as, for instance, a two dimensional Gaussian used elsewhere [15]), with density large in the center and decreasing outwards, will be deformed to a cross-section with a horse-shoe shape [20] as it expands by being accelerated in the direction of the major radius of the toroid, here the $\widehat{\mathbf{R}}$-direction.

While the blob moves in the positive $R$-direction (i.e. the direction of decreasing magnetic field) also its average density decreases since the net integrated plasma in the cylindrical volume is conserved. The radius in the "dough-nut" increases while its small radius is constant so $n \sim 1 / R$ just like $B \sim 1 / R$. This density variation is small for relevant cases, but it is easy to account for as long as we at any time can take the density to be constant in a cross section.

The simple model assumed a circular plasma cross section with uniform density. The spatial toroidal magnetic field variation was included by retaining a $\nabla B$-drift of ions and electrons, assuming the magnetic field to be constant otherwise. The model 
is self-consistent since a circular plasma column with uniform density will retain its circular cross-section for a spatially constant $\nabla B$-drift velocity.

The $\nabla B$-drift polarizes the blob and induces an $m=1$ mode on the potential variation. This is a basic mode of perturbation, originating from the fact that the plasma does not have a simple steady state toroidal equilibrium [21]. The corresponding homogeneous electric field variation has the direction $-\boldsymbol{\Delta}(t)$. Within this simple model, the electrostatic potential fluctuations within the plasma blob are in phase for all $R$ positions and fixed $z$, see Figs. 1 and 2.
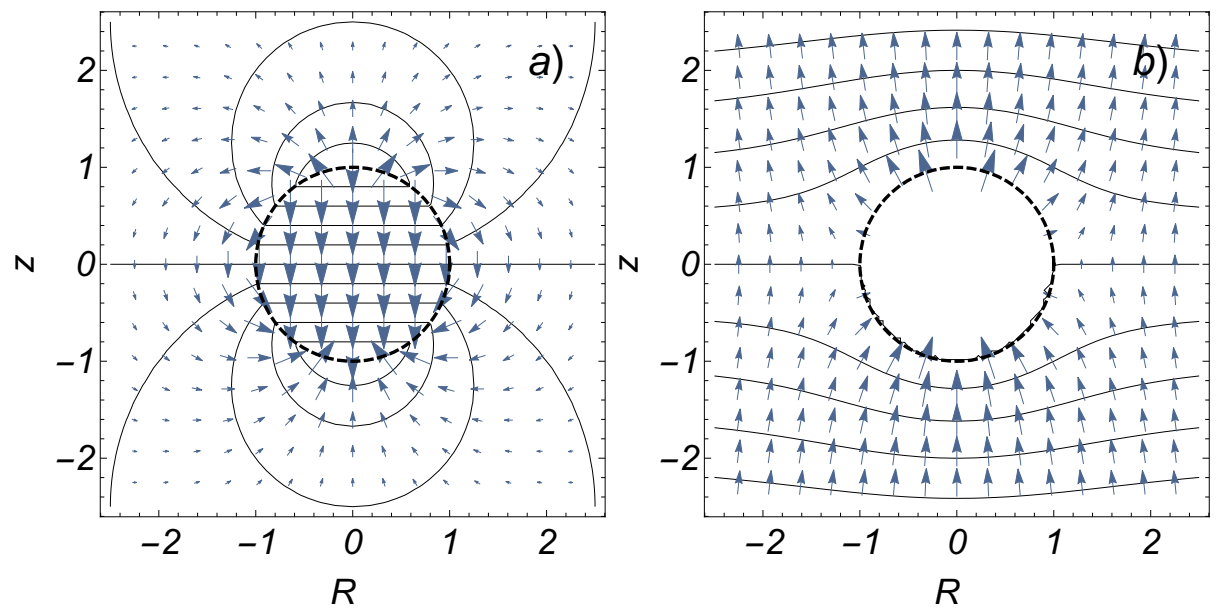

Figure 2. Illustration of the electric fields and equi-potential lines for the simple polarized top-hat model in the fixed laboratory frame shown in a). The dashed circle gives the boundary of the top-hat blob density variation. The blob radius is here $R_{b}=1$. In $\mathrm{b}$ ) we show the flow lines in a co-moving frame of reference, assuming here the local magnetic field to be homogeneous so that the $\mathbf{E} \times \mathbf{B} / B^{2}$-flow in incompressible. Positions $R$ are measured here with respect to the reference position $R_{0}$.

In some toroidal experiments inward propagating density depletions have been observed [12]. Such phenomena can quantitatively also be accounted for by a generalization of the foregoing model. We here thus assume the density depletion to have a top-hat form with depth $n_{1}$ in a plasma background of density $n_{0} \geq n_{1}$. Many results can be found by simple generalization of those from the previous subsection by introducing a negative density perturbation associated with the blob.

\section{Applications to plasmas in gravitational fields}

The foregoing results can be applied for plasmas near equator, where the gravitational field is approximately perpendicular to the magnetic fields. The magnetic field can here be taken homogeneous, but the gravitational field gives a polarization very much like the $\nabla B$-drift in the foregoing analysis. With $\mathbf{g} \perp \mathbf{B}$ being the gravitational acceleration, we have $\mathbf{U}_{i}=M \mathbf{g} \times \mathbf{B} /\left(q B^{2}\right)$. The results for the present problem can then be obtained by using $U_{i}=g / \Omega_{c i}$, while $U_{e} \approx 0$ because of the smallness of the gravitational force on electrons. The expression for the acceleration becomes particularly simple [20] in 
the limit of high density where $\epsilon_{r} \sim n_{0}$, i.e. $d^{2} \mathbf{R}_{p} / d t^{2}=\Omega_{c i}\left(U_{i}+U_{e}\right) \widehat{\mathbf{g}}=\mathbf{g}$ by use of (9) for the present conditions with the direction of gravity replacing the $\nabla B$-direction. A plasma blob at high density in a gravitational field will drop like a brick when it is infinitely elongated along a homogeneous horizontal magnetic field. The acceleration becomes gradually smaller as the density is decreased, and ultimately as $n_{0} \rightarrow 0$ we find an electron-ion pair drifting in opposite directions due to their respective $\mathbf{g} \times \mathbf{B}$-drifts.

Solar coronal loops or solar prominences can be kept floating by the gradient in magnetic pressure that results from the curvature of the magnetic fields [16]. This pressure force counteracts gravity. The plasma drifts caused by gravity and $\nabla B$-drifts balance each other, at least partially. When the magnetic field lines are bent, the plasma can flow in the vertical direction along $\mathbf{B}$ under the influence of gravity, and other effects can have a role here [23]. The magnetic curvature affects both electrons and ions as long as their temperatures are comparable, while gravity acts mostly on the heavy ion component. An approximate balance can be argued when $\bar{M} g /(e B) \approx U_{i}+U_{e} \approx 2 U_{i}$ with $\bar{M}$ being an average ion mass and $U_{i}$ being the ion $\nabla B$-drift. We again estimate $|\nabla B| \approx B / R_{c}$ with $R_{c}$ here being the local radius of curvature of the magnetic field lines [16], and $g \approx G M_{\odot} / R_{\odot}^{2}$ with $G=6.67 \times 10^{-11} \mathrm{~N} \mathrm{~m}^{2} \mathrm{~kg}^{-2}$ being the gravitational constant, $M_{\odot} \approx 1.99 \times 10^{30} \mathrm{~kg}$ being the solar mass and $R_{\odot} \approx 6.96 \times 10^{8} \mathrm{~m}$ being the average solar radius. An approximate balance giving equilibrium between gravity and magnetic gradient drifts is then found by $(G / e) \bar{M} M_{\odot} /\left(B R_{\odot}^{2}\right) \approx T /\left(e B R_{c}\right)$, or $R_{c} \approx T R_{\odot}^{2} /\left(G \bar{M} M_{\odot}\right)$ with $T$ being an average plasma temperature. The result is independent of the magnetic field and the plasma density. With typical parameters we find as an order of magnitude the balance for $R_{c} \approx 10^{6}-10^{7} \mathrm{~m} \ll R_{\odot}$. Smaller curvature radii gives a strong magnetic pressure gradient that erupts the protuberance, while for larger curvature radii the magnetic field pressure gradient can no longer support the plasma blob against gravity. Gradients in plasma temperature are not considered here, but in order to have an effect, their scale lengths must be comparable to the blob diameter.

For application for the Earth's ionosphere in the equatorial region we can consider a different formulation of the present problem. Here the vertical motion of "bubbles" is frequently observed [24]. The fluctuations in plasma density can be seen as depletions or "bite-outs" of the background plasma density in a horizontal magnetic flux tube. The bubbles are here the saturated stage of the Rayleigh-Taylor instability excited in the bottom region of the equatorial ionosphere [25]. We can model such a density depletion by assigning a negative density $-n_{0}$ to the blob in our expressions, where it is then implicitly assumed that surrounding background plasma has a density exceeding $n_{0}$. Consequently we find in our case a constant vertical acceleration of the bubbles towards higher altitudes. This acceleration will be reduced by viscosity and the drag due to collisions between plasma particles and neutrals. 


\section{Extensions of the model}

The model has some generalizations, the simplest one consisting of an approximation to a continuous distribution by use of several "steps" in density as illustrated in Fig. 3, here with only two steps. The motion of the individual layers can be attributed to basically two effects. One is the self-induced motion that depends on the density enhancement. This effect has been discussed already. It implies that the largest density blob moves fastest, the other successively slower as also illustrated in Fig. 3. The other effect is due to the distortion of the selected level by all the other density levels. We illustrate this latter case here. As a first approximation we can let the lowest density part in Fig. 3 with radius $R_{b}=1.5$ be passively convected by the velocity field induced by the inner higher density core, here with radius $R_{b}=1$, with the velocity vectors as shown in Fig. 2b). One immediate observation from this simple calculation is the steepening of the plasma density gradient at the stagnation point in agreement with previous results $[15]$.
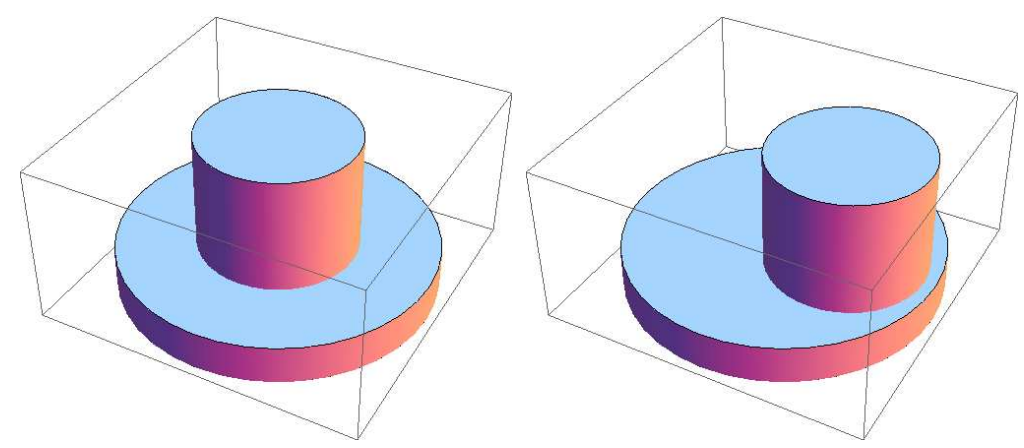

Figure 3. Schematic illustration of blob-density distributions composed of several "steps" in density, here shown for 2 steps. The figure to the left is the initial condition, which with time distorts to the right in the limit where the interaction between the two density levels is ignored and each one propagates by its own induced polarization field.

\section{Modifications of the ion and electron dynamics}

The foregoing basic discussion assumed the bulk motion of both electrons and ions to be in the direction perpendicular to the magnetic field. For plasmas with a toroidal magnetic transform, the model needs to be amended. While the equation for the low frequency ion dynamics can be assumed to be relatively general, the corresponding expression for the electrons is restrictive by not including the effect of electrons moving along the magnetic field lines due to a small vertical $\mathbf{B}$-field component. To simplify the analysis we use a locally cylindrical model where the magnetic field has a small vertical component, which allows for a vertical component of the electron motion that to counteracts the $\nabla B$-drift. The polarization charges that give the electric field along the $\Delta$-direction are then reduced. 


\subsection{Effects of a small vertical magnetic field component}

The first modification of the basic model assumes that the electric field along the tilted magnetic field lines is constant and given as $E_{b}=E_{z} \sin \theta \equiv \mathbf{E} \cdot \widehat{\mathbf{z}} \sin \theta \approx \mathbf{E} \cdot \widehat{\mathbf{z}} \theta$ where $\theta$ is the angle between the toroid axis (the $x$-axis in Fig. 1) and the slightly tilted magnetic field vector B. The present model can be seen as a local representation for a toroidal transform of the magnetic field. We allowed for the possibility that $\mathbf{E}$ need not be strictly along $\widehat{\mathbf{z}}$. By the present model we in effect assume the electron collisional mean free path to be smaller than the length scale of one turn in the toroidal transform. To describe the electron motion with collisions we can then use

$$
0 \approx-T_{e} \frac{\partial n}{\partial s}-n e E_{b}-n m \nu U_{e b}
$$

where $s$ is the coordinate along the tilted magnetic field lines, and the subscript $b$ specifies electric field and electron fluid velocity components along $\mathbf{B}$. We introduced $\nu$ as an electron collision frequency and $T_{e}$ as a constant electron temperature. Electron inertia has been ignored due to the smallness of the electron mass $m$,

\subsubsection{Boltzmann distributed electrons The simplest case where the electrons flow freely} (i.e. with $\nu \approx 0$ in (10)) to maintain an isothermal Boltzmann equilibrium that gives $n / n_{0} \approx e \phi / T_{e}$ with $n_{0}$ being some reference density. This limit corresponds to the one used for deriving the Hasegawa-Mima equation [26]. For the top-hat model we will have a constant potential inside the circular contour confining the blob and the electric field vanishes there. At the edge of the structure we find a radial electric field which in this case gives rise to an $\mathbf{E} \times \mathbf{B} / B^{2}$-rotation of a thin surface layer. The net blob displacement will be solely due to the ion $\nabla B$-drift in this limit. The assumption of Boltzmann distributed electrons ignores electron inertia. Retaining a non-vanishing electron mass will give a short delay which allows for a weak vertical electric field to develop inside the structure. For realistic applications of the analysis, the effects of electron inertia are found to be immaterial.

\subsubsection{Constant electron mobility A non-vanishing collision frequency $\nu$ in (10) gives} rise to a delay that resembles the effects of electron inertia, although it contributes with a different phase in the time variation. Within the top-hat model we have the plasma density to be constant and find $U_{e b} \approx-e E_{b} /(\nu m)$ giving $U_{e z} \approx U_{e b} \sin \theta \approx$ $-(e / \nu m) E_{b} \sin \theta \approx-(e / \nu m) E_{z} \theta^{2}=-(e / \nu m) \mathbf{E} \cdot \widehat{\mathbf{z}} \theta^{2}$ corresponding to a motion with constant electron mobility. For weak collisionality, small $\nu$, we have $U_{e z}$ to be the dominant electron velocity having a vertical component in the $\widehat{\mathbf{z}}$-direction: even though $\theta$ is small, this velocity component can be large due to the smallness of $\nu$. This velocity is now assumed to dominate the $\nabla B$ electron drift in the $\widehat{\mathbf{z}}$-direction.

The electron equation of motion becomes

$$
\frac{d}{d t} \mathbf{R}_{e}(t)=\frac{\mathbf{E}\left(\mathbf{R}_{e}(t), t\right) \times \mathbf{B}}{B^{2}}-\frac{e}{\nu m} \mathbf{E}\left(\mathbf{R}_{e}(t), t\right) \cdot \widehat{\mathbf{z}} \theta^{2} \widehat{\mathbf{z}},
$$


instead of (3). We still have $\mathbf{E}=-\frac{1}{2}\left(e n_{0} / \varepsilon_{0}\right) \boldsymbol{\Delta}$, giving

$$
\frac{d}{d t} \mathbf{R}_{e}(t)=-\frac{1}{2} \frac{e n_{0}}{\varepsilon_{0} B^{2}} \boldsymbol{\Delta} \times \mathbf{B}+\frac{1}{2} \frac{e^{2} n_{0}}{\varepsilon_{0} m \nu} \boldsymbol{\Delta} \cdot \widehat{\mathbf{z}} \theta^{2} \widehat{\mathbf{z}} .
$$

For the ion dynamics we ignore collisions and have the previous result

$$
\frac{d}{d t} \mathbf{R}_{i}(t)=-\frac{1}{2} \frac{e n_{0}}{\varepsilon_{0} B^{2}} \boldsymbol{\Delta} \times \mathbf{B}-\frac{1}{2} \frac{e n_{0}}{\varepsilon_{0} B \Omega_{c i}} \frac{d \boldsymbol{\Delta}}{d t}+U_{i} \widehat{\mathbf{z}} .
$$

We have for $\boldsymbol{\Delta}(t) \equiv \mathbf{R}_{i}(t)-\mathbf{R}_{e}(t)$ the first order differential equation

$$
\left(1+\frac{1}{2} \frac{\Omega_{p i}^{2}}{\Omega_{c i}^{2}}\right) \frac{d \boldsymbol{\Delta}}{d t}=-\frac{1}{2} \frac{\omega_{p e}^{2}}{\nu} \theta^{2}(\boldsymbol{\Delta} \cdot \widehat{\mathbf{z}}) \widehat{\mathbf{z}}+U_{i} \widehat{\mathbf{z}} .
$$

Taking the scalar product $\widehat{\mathbf{z}} \cdot$ of all terms we readily find

$$
\frac{1}{2}\left(1+\epsilon_{r}\right) \frac{d(\boldsymbol{\Delta} \cdot \widehat{\mathbf{z}})}{d t}=-\frac{1}{2} \frac{\omega_{p e}^{2}}{\nu} \theta^{2}(\boldsymbol{\Delta} \cdot \widehat{\mathbf{z}})+U_{i}
$$

which has simple solutions with $U_{i}$ constant. Making a local model, we take also $\Omega_{p i}^{2}$ and $\Omega_{c i}^{2}$ to be constant. The solution is then

$$
\boldsymbol{\Delta} \cdot \widehat{\mathbf{z}}=2 \frac{U_{i} \nu}{\theta^{2} \omega_{p e}^{2}}+C_{1} \exp \left(-\frac{t}{1+\epsilon_{r}} \frac{\omega_{p e}^{2}}{\nu} \theta^{2}\right),
$$

with $C_{1}$ being an integration constant. The result demonstrates that the component of the polarization $\boldsymbol{\Delta}$ in the $\widehat{\mathbf{z}}$-direction eventually reaches a constant level due to the short-circuiting effect of electron motion along magnetic field lines. Inserting (14) into (15) we find that $\boldsymbol{\Delta}$ itself approaches a constant value. The characteristic time for reaching this saturated stage is is $\nu\left(1+\epsilon_{r}\right) \omega_{p e}^{-2} \theta^{-2}$ which varies with density but not with plasma temperature. The interesting feature is here that the saturation time is not determined solely by $\nu$.

For $\mathbf{R}_{p}(t) \equiv \frac{1}{2}\left(\mathbf{R}_{i}(t)+\mathbf{R}_{e}(t)\right)$ we find

$$
\frac{d}{d t} \mathbf{R}_{p}(t)=-\frac{e n_{0}}{2 \varepsilon_{0} B^{2}} \boldsymbol{\Delta} \times \mathbf{B}-\frac{1}{2} \frac{e n_{0}}{\varepsilon_{0} B \Omega_{c i}} \frac{d \boldsymbol{\Delta}}{d t}+\frac{1}{4} \frac{e^{2} n_{0}}{\varepsilon_{0} m \nu} \theta^{2}(\boldsymbol{\Delta} \cdot \widehat{\mathbf{z}}) \widehat{\mathbf{z}}+\frac{U_{i}}{2} \widehat{\mathbf{z}},
$$

where we insert the solution found for $\boldsymbol{\Delta}(t)$. The two last terms sum up to $U_{i} \widehat{\mathbf{z}}$ in the limit of large $t$. The term with $d \boldsymbol{\Delta} / d t$ vanishes in the same limit. For large $t$, the first term on the right hand side becomes $U_{i}\left(\Omega_{p i} / \Omega_{c i}\right)^{2} \theta^{-2}\left(\nu / \omega_{p e}^{2}\right) \widehat{\mathbf{R}}$. The blob will perform an oblique orbit in the $(x, y)$-plane in this limit. With $\boldsymbol{\Delta}(t)$ asymptotically constant, the blob will move with constant velocity as $t \rightarrow \infty$, i.e. without acceleration in contrast to the case where electron motion along magnetic field lines is ignored. The asymptotic velocity depends critically on the angle $\theta$. Note that the assumption (10) is invalidated when $\theta \rightarrow 0$, so this limit can not be applied in (15).

If we initiate a plasma blob that is strictly charge neutral (i.e. not merely quasi neutral [16]) with $\boldsymbol{\Delta}=0$, the ion polarization via $U_{i}$ will induce an electric field in the blob and set it into motion. Its velocity will increase until it reaches an asymptotic level given before. 


\subsection{Ion friction through neutral collisions}

Another extension of the model is found by including also ion neutral collisions with frequency $\nu_{i}$. In this case we modify the ion dynamics by rewriting (13) to include a collisional friction in the analytical form

$$
\frac{d^{2}}{d t^{2}} \mathbf{R}_{i}(t)=-\frac{1}{2} \frac{e n_{0}}{\varepsilon_{0} B^{2}} \frac{d \boldsymbol{\Delta}}{d t} \times \mathbf{B}-\frac{1}{2} \frac{e n_{0}}{\varepsilon_{0} B \Omega_{c i}} \frac{d^{2} \boldsymbol{\Delta}}{d t^{2}}-\nu_{i} \frac{d}{d t} \mathbf{R}_{i}(t) .
$$

With $\mathbf{R}_{i}=\boldsymbol{\Delta}+\mathbf{R}_{e}$ we have

$$
\begin{aligned}
& \frac{d^{2}}{d t^{2}} \boldsymbol{\Delta}(t)+\frac{d^{2}}{d t^{2}} \mathbf{R}_{e}(t)=-\frac{1}{2} \frac{e n_{0}}{\varepsilon_{0} B^{2}} \frac{d \boldsymbol{\Delta}}{d t} \times \mathbf{B} \\
& -\frac{1}{2} \frac{e n_{0}}{\varepsilon_{0} B \Omega_{c i}} \frac{d^{2} \boldsymbol{\Delta}}{d t^{2}}-\nu_{i} \frac{d}{d t} \boldsymbol{\Delta}(t)-\nu_{i} \frac{d}{d t} \mathbf{R}_{e}(t),
\end{aligned}
$$

where we insert $d \mathbf{R}_{e}(t) / d t$ from (12) to find

$$
\begin{aligned}
& \frac{1}{2}\left(1+\varepsilon_{r}\right) \frac{d^{2}}{d t^{2}} \boldsymbol{\Delta}(t)+\frac{1}{2} \frac{e^{2} n_{0}}{\varepsilon_{0} m \nu} \frac{d \boldsymbol{\Delta} \cdot \widehat{\mathbf{z}}}{d t} \theta^{2} \widehat{\mathbf{z}}= \\
& -\nu_{i} \frac{d}{d t} \boldsymbol{\Delta}(t)+\frac{1}{2} \frac{\nu_{i} e n_{0}}{\varepsilon_{0} B^{2}} \boldsymbol{\Delta} \times \mathbf{B}-\frac{1}{2} \frac{e^{2} n_{0} \nu_{i}}{\varepsilon_{0} m \nu} \boldsymbol{\Delta} \cdot \widehat{\mathbf{z}} \theta^{2} \widehat{\mathbf{z}} .
\end{aligned}
$$

A stationary asymptotic solution for $\Delta$ is found if and only if

$$
\frac{1}{B} \boldsymbol{\Delta} \times \mathbf{B}=\frac{\omega_{c e}}{\nu}(\boldsymbol{\Delta} \cdot \widehat{\mathbf{z}}) \theta^{2} \widehat{\mathbf{z}}
$$

This result imposes $\boldsymbol{\Delta} \times \mathbf{B} \| \widehat{\mathbf{z}}$ and thereby $\boldsymbol{\Delta} \| \widehat{\mathbf{R}}$ also for $\theta \neq 0$. It is then readily seen that (19) has no solution for any vector $\boldsymbol{\Delta} \neq 0$. The asymptotic stationary solution where $\boldsymbol{\Delta}=0$ means that the blob reaches "halt". By (16) we argue that a characteristic time for arresting the blob motion is $\nu_{i}^{-1}$. The expression (18) can be separated into vector components and solved in detail to give the entire time variation of $\boldsymbol{\Delta}(t)$. The present result deserves scrutiny in light of experimental observations where the blob velocity seems only weakly affected by ion-neutral collisions [11].

If we initiate a plasma blob that is strictly charge neutral, $\boldsymbol{\Delta}(t=0)=0$ with the additional constraint $d \boldsymbol{\Delta} /\left.d t\right|_{t=0}=0$, it will remain so and there will be no net displacement of the blob. The present analysis retains a "top-hat" model even with ionneutral collisions included. In reality, collisional diffusion will smear out this idealized density variation with time.

\section{Consequences of compressible flows}

The analysis so far uses the approximation $\nabla \cdot\left(\mathbf{E} \times \mathbf{B} / B^{2}\right) \approx 0$ for electrostatic conditions. This remains correct as long as we can assume $\mathbf{B} \approx$ constant, as in Fig. $2 \mathrm{~b}$ ). Concerning the $\nabla B \times \mathbf{B}$-drift we used the standard approximation of a magnetic field varying linearly with the radial variable as $\mathbf{B}=\left\{0,0, B_{0}\left(R_{0}\right) /\left(1+R / R_{0}\right)\right\}$ where $R_{0}$ is a reference position at the center of the circular cross section of the torus, with $R / R_{0}$ here being a small quantity, the direction of $R$ explained in Fig. 1 . With this approximation the intensity of the magnetic field is spatially varying, but we let the 
direction be constant. Allowing for spatial variations of the magnetic field we can modify the $\mathbf{u}_{E \times B}=\mathbf{E} \times \mathbf{B} / B^{2}$-velocities in the previous sections by taking

$$
\mathbf{u}_{E \times B} \approx \frac{\mathbf{E} \times \mathbf{B}_{0}}{B_{0}^{2}}\left(1+\frac{R}{R_{0}}\right) .
$$

Within the present model we have $\nabla \cdot \mathbf{u}_{E \times B}=\mathbf{E} \times \mathbf{B}_{0} \cdot \widehat{\mathbf{R}} /\left(R_{0} B_{0}^{2}\right) \approx E /\left(R_{0} B_{0}\right)$ which will be useful later on. Note that $\nabla \cdot \mathbf{u}_{E \times B}$ is here the same in a fixed or a moving frame of reference.

\subsection{Isolated blobs}

With the approximation (20) we have slightly different velocities of the high and low magnetic field-sides of the blob with initially circular cross section. At later times the blob will obtain an elliptic cross section with a major axis that increases linearly with time. The minor axis will remain constant. The density $n_{0}$ in the initial "top-hat" will decrease with time but remain spatially constant inside the ellipse in such a way that $n_{0}(t)$ multiplied with the area of the ellipse remains constant in time. As the ellipse becomes elongated the factor $1 / 2$ in (4) is changed and in the limit of a very long ellipse we have $1 / 2 \rightarrow 1$ as appropriate for a slab geometry. This effect tends to increase $E$. On the other hand the decreasing density $n_{0}$ compensates this effect and we have $\mathbf{u}_{E \times B}$ to remain approximately constant. If the initial density $n_{0}(0)$ is sufficiently large to allow the saturation approximation $\varepsilon_{r} \equiv 1+n_{0}(0) M / \varepsilon_{0} B_{0}^{2} \approx n_{0}(0) M / \varepsilon_{0} B_{0}^{2}$ we can assume the approximation to remain valid for some time and the change in plasma density inside the elliptical contour has only little consequence, having in mind also that the blob will arrive at the wall of the confining plasma vessel in a relatively short time. For small initial plasma densities in the blob the conclusion has to be modified, and the density variation will here have comparatively smaller effect meaning that the increase in electric field (4) will be somewhat more important. We can conclude that for an isolated blob, the consequences of compressible flows due to spatially varying magnetic fields will generally be of little consequence.

\subsection{Blobs embedded in a plasma background}

For a blob propagating in a plasma background the changes in the flow velocities induced by the blob in the surrounding plasma need to be accounted for. If the background is initially homogeneous, then a moving blob will induce compressible motions and density perturbations in its surroundings. Taking Figs. 2a) and 2b) as reference we note that the $\mathbf{E} \times \mathbf{B} / B^{2}$-velocities induced in the surrounding plasma by the blob at $R>R_{0}$ will be larger than at $R_{0}$, while at the symmetric position for $R<R_{0}$ the velocity will be smaller. Starting with an initially homogeneous plasma we have from the plasma continuity equation $\partial n / \partial t \approx-n \nabla \cdot \mathbf{u}_{E \times B} \approx-n E /\left(R_{0} B_{0}\right) \sim-n E / B_{0}$. Since $n>0$ always, the sign of the rate of change in the plasma density as induced by the compressible flow around the blob is then given solely by the sign of $-E / B_{0}$. With 
reference to Fig. 2 (where $B_{0}<0$ ) we expect a density depletion to form along the $R$ axis, while the plasma density will be enhanced on the top and bottom sides (measured along the $z$-direction) of the plasma blob.

\section{Conclusions}

In the present study we analyzed a simple but solvable blob-model. The model has a number of basic results. For the strictly magnetic field aligned plasma blob, where both the ion and electron bulk motion is perpendicular to $\mathbf{B}$, we find a constant radial acceleration of the blob, in the major radius direction of a toroid. The value acceleration reaches a constant level as the plasma density is increased to have $\Omega_{p i} \gg \Omega_{c i}$. For reduced densities the acceleration is correspondingly reduced. The model assumes that the blob radius $R_{b}$ is much larger than the ion Larmor radius $r_{L i}$. For smaller $R_{b}$, the finite ion Larmor radius effects will average the spatial variations of the electric fields and thereby reduce the blob acceleration $[27,28]$. As an order of magnitude estimate $[16,17]$ we can account for this effect by introducing a reduction factor $\left(1-r_{L i}^{2} / R_{b}^{2}\right)$ on the electric fields and thereby on the velocity. Formally, the model allows for large spatial separations of the electron and ion components. This unphysical limit will however have little practical consequence since it gives very large $\mathbf{E} \times \mathbf{B} / B^{2}$-velocities, and the plasma will be rapidly lost to the confining walls of the plasma.

We illustrated how electron motion along magnetic field lines will partially shortcircuit the polarization electric fields to give an asymptotically constant blob velocity which scales as $\sim \nu T$, where the temperature $T$ scaling originates from $U_{i}$. Since $\varepsilon_{r}$ disappears from the asymptotic result, there is here no dependence on the plasma density associated with the blob.

The basic simplification of the model lies in an assumption of a constant density in the cross section. It is feasible to make an approximation to a multiple top-hat density distribution, with density "steps" in the cross section of the blob. For numerical modeling this approach has an advantage that it suffices to follow a small number of contours rather than the entire plasma density variation. In studies of neutral flows this approach was advantageous [29]. In that case, however, the tracer material was passively convected, while in the present plasma equivalent of the problem the contours are mutually interacting through the collective electric fields. The general analysis has elements in common with studies of "MHD-droplets", but these more general cases include also viscous drags from the surrounding fluid [30]. An enhancement in plasma density, or an isolated localized blob of plasma, can propagate due to induced electric fields caused by charge separations generated by particle drifts. Similarly we can describe a localized depletion in an otherwise uniform plasma by a very similar analysis. Such cases have relevance for instance in modeling of Rayleigh-Taylor bubbles in the equatorial ionosphere $[24,25]$. Blob propagation for conditions where we have electron and ion drifts perpendicular to the magnetic field in collisional parts of the lower ionosphere have interesting properties [31], but these problems are not considered 
here.

The analysis presented in this work deals with isolated blobs, possibly embedded in a background plasma. Two close blobs can interact presumably the same way as convective cells [32]. The spatial variations of the flow distributions shown in Fig. 2 can be used as a guide for this process.

\section{Acknowledgments}

This work was supported by the Research Council of Norway under grant 240510/F20.

\section{References}

[1] O. G. Bakunin. Turbulence and Diffusion: Scaling Versus Equations. Springer Series in Synergetics. Springer, Berlin, 2008.

[2] W. Horton. Nonlinear drift waves and transport in magnetized plasma. Phys. Reports, 192:1 177, 1990. doi:10.1016/0370-1573(90)90148-U.

[3] A. H. Nielsen, H. L. Pécseli, and J. Juul Rasmussen. Turbulent transport in low- $\beta$ plasmas. Phys. Plasmas, 3:1530-1544, 1996. doi:10.1063/1.872008.

[4] T. Yamada, S-I. Itoh, T. Maruta, N. Kasuya, Y. Nagashima, S. Shinohara, K. Terasaka, M. Yagi, S. Inagaki, Y. Kawai, A. Fujisawa, and K. Itoh. Anatomy of plasma turbulence. Nature Phys., 4:721-725, 2008. doi:10.1038/nphys1029.

[5] A. S. Bergsaker, A. Fredriksen, H. L. Pécseli, and J. K. Trulsen. Models for the probability densities of the turbulent plasma flux in magnetized plasmas. Physica Scripta, 90:108005, 2015. doi:10.1088/0031-8949/90/10/108005.

[6] O. E. Garcia, N. H. Bian, V. Naulin, A. H. Nielsen, and J. Juul Rasmussen. Mechanism and scaling for convection of isolated structures in nonuniformly magnetized plasmas. Phys. Plasmas, 12:090701, 2005. doi:10.1063/1.2044487.

[7] O. Grulke, J. L. Terry, B. LaBombard, and S. J. Zweben. Radially propagating fluctuation structures in the scrape-off layer of Alcator C-Mod. Phys. Plasmas, 13:012306, 2005. doi:10.1063/1.2164991.

[8] I. Furno, B. Labit, A. Fasoli, F. M. Poli, P. Ricci, C. Theiler, S. Brunner, A. Diallo, J. P. Graves, M. Podestà, and S. H. Müller. Mechanism for blob generation in the TORPEX toroidal plasma. Phys. Plasmas, 15:055903, 2008. doi:10.1063/1.2870082.

[9] I. Furno, B. Labit, M. Podestà, A. Fasoli, S. H. Mueller, F. M. Poli, P. Ricci, C. Theiler, S. Brunner, A. Diallo, and J. Graves. Experimental observation of the blob-generation mechanism from interchange waves in a plasma. Phys. Rev. Lett., 100:055004, 2008. doi:10.1103/PhysRevLett.100.055004.

[10] S. H. Müller, C. Theiler, A. Fasoli, I. Furno, B. Labit, G. R. Tynan, M. Xu, Z. Yan, and J. H. Yu. Studies of blob formation, propagation and transport mechanisms in basic experimental plasmas (TORPEX and CSDX). Plasma Phys.Contr. Fusion, 51:055020, 2009. doi:10.1088/0741$3335 / 51 / 5 / 055020$.

[11] C. Theiler, I. Furno, P. Ricci, A. Fasoli, B. Labit, S. H. Müller, and G. Plyushchev. Crossfield motion of plasma blobs in an open magnetic field line configuration. Phys. Rev. Lett., 103:065001, 2009. doi:10.1103/PhysRevLett.103.065001.

[12] J. Cheng, L. W. Yan, W. Y. Hong, K. J. Zhao, T. Lan, J. Qian, A. D. Liu, H. L. Zhao, Y. Liu, Q. W. Yang, J. Q. Dong, X. R. Duan, and Y. Liu. Statistical characterization of blob turbulence across the separatrix in HL-2A tokamak. Plasma Phys. Control. Fusion, 52:055003, 2010. doi:10.1088/0741-3335/52/5/055003.

[13] D. A. D'Ippolito, J. R. Myra, and S. J. Zweben. Convective transport by intermittent 
blob-filaments: comparison of theory and experiment. Phys. Plasmas, 18:060501, 2011. doi:10.1063/1.3594609.

[14] B. Labit, C. Theiler, A. Fasoli, I. Furno, and P. Ricci. Blob-induced toroidal momentum transport in simple magnetized plasmas. Phys. Plasmas, 18:032308, 2011. doi:10.1063/1.3559462.

[15] R. Kube and O. E. Garcia. Velocity scaling for filament motion in scrape-off layer plasmas. Phys. Plasmas, 18:102314, 2011. doi:10.1063/1.3647553.

[16] F. F. Chen. Introduction to Plasma Physics and Controlled Fusion, volume 1. Plenum Press, New York, 2 edition, 1984.

[17] G. Knorr, F. R. Hansen, J. P. Lynov, H. L. Pécseli, and J. J. Rasmussen. Finite Larmor radius effects to arbitrary order. Phys. Scripta, 38:829-834, 1988. doi:10.1088/0031-8949/38/6/014.

[18] A. Hasegawa and M. Wakatani. Plasma edge turbulence. Phys. Rev. Lett., 50:682-686, 1983. doi:10.1103/PhysRevLett.50.682.

[19] O. E. Garcia. Collective motions in non-uniformly magnetized plasmas. European J. Phys., 24:331-339, 2003. doi:10.1088/0143-0807/24/4/351.

[20] S. Chandrasekhar. Plasma Physics. The University of Chicago Press, Chicago, 1960. Notes compiled by S. K. Trehan after a course given by S. Chandrasekhar.

[21] K. Rypdal, E. Grønvoll, F. Øynes, Å. Fredriksen, R. J. Armstrong, J. Trulsen, and H. L. Pécseli. Confinement and turbulent transport in a plasma torus with no rotational transform. Plasma Phys. Contr. Fusion, 36:1099-1114, 1994. doi:10.1088/0741-3335/36/7/002.

[22] K. Rypdal, O. E. Garcia, and J.-V. Paulsen. Anomalous cross-field current and fluctuating equilibrium of magnetized plasmas. Phys. Rev. Lett., 79:1857-1860, 1997. doi:10.1103/PhysRevLett.79.1857.

[23] H. L. Pécseli and O. Engvold. Modeling of prominence threads in magnetic fields: levitation by incompressible MHD waves. Solar Phys., 194:73-86, 2000. doi:10.1023/A:1005242609261.

[24] R. F. Woodman. Spread F - an old equatorial aeronomy problem finally resolved? Ann. Geophysicae, 27:1915-1934, 2009. doi:10.5194/angeo-27-1915-2009.

[25] E. Ott. Theory of Rayleigh-Taylor bubbles in the equatorial ionosphere. J. Geophys. Res., 83:2066-2070, 1978. doi:10.1029/JA083iA05p02066.

[26] A. Hasegawa and K. Mima. Pseudo-three-dimensional turbulence in magnetized nonuniform plasma. Phys. Fluids, 21:87-92, 1978. doi:10.1063/1.862083.

[27] J. Madsen, O. E. Garcia, J. Stærk Larsen, V. Naulin, A. H. Nielsen, and J. J. Rasmussen. The influence of finite larmor radius effects on the radial interchange motions of plasma filaments. Phys. Plasmas, 18:112504, 2011. doi:10.1063/1.3658033.

[28] M. Wiesenberger, J. Madsen, and A. Kendl. Radial convection of finite ion temperature, high amplitude plasma blobs. Phys. Plasmas, 21:092301, 2014. doi:10.1063/1.4894220.

[29] B. Krane, H. L. Pécseli, and J. Trulsen. Concentrations and concentration fluctuations in twodimensional turbulence. Phys. Fluids, 15:211-226, 2003. doi:10.1063/1.1524628.

[30] J. P. Narain and M. S. Uberoi. Magnetohydrodynamics of a drop. Phys. Fluids, 15:62-69, 1972. doi:10.1063/1.1693756.

[31] P. Høeg. Directional changes in the irregularity drift during artificial generation of striations. Phys. Scripta, 33:469-474, 1986. doi:10.1088/0031-8949/33/5/016.

[32] H. L. Pécseli, J. Juul Rasmussen, and K. Thomsen. Nonlinear interaction of convective cells in plasmas. Plasma Phys. Contr. Fusion, 27:837-846, 1985. doi:10.1016/0741-3335/27/8/002. 